\title{
Combining Deep Learning Technologies with Multi-Level Gabor Features for Facial Recognition in Biometric Automated Systems
}

\author{
Catalin-Mircea DUMITRESCU*, Ioan DUMITRACHE \\ University POLITEHNICA of Bucharest, 313 Splaiul Independenței, Bucharest, Romania \\ dumitrescu.catalin.m@gmail.com (*Corresponding author), ioan.dumitrache@acse.pub.ro
}

\begin{abstract}
Face recognition is one of the most important abilities that humans use in their daily lives. It represents a natural, robust and non-intrusive manner for identifying individuals. However, it is also a very challenging problem in the field of computer vision and pattern recognition. A good face recognition algorithm should be able to automatically detect and recognize a face in an image, regardless of lightning, expression, illumination and pose. In this paper, we present a novel approach for the face model representation and matching issues in face recognition. Our approach is based on multi-level Gabor features and Deep Learning techniques. In the experiments presented in this paper, ORL, Caltech, Yale and Yale B databases were used in order to obtain the face recognition rate. The results show that the new face recognition algorithm outperforms the conventional methods such as global Gabor face recognition based on PCA in terms of recognition rate.
\end{abstract}

Keywords: Face Recognition, Multi-level Gabor features, Diabolo Networks, Auto-encoders, ORL (Olivetti Research Laboratory).

\section{Introduction}

A biometric marker (trait) can be defined as an anatomic attribute (face, fingerprint, DNA, iris etc.) or behavioural characteristic (voice, signature etc.), which can be used alone, or in combination with other traits, in order to identify / recognize a person, a prerequisite in many applications such as control, defence, banking and so on.

The decrease in the production costs of biometric sensors, combined with the advances registered by Information and Communication Technology (ICT) and mobile devices, stimulated the research and development of Automated Biometric Recognition Systems (ABRS).

Thus, according to Acuity Market Intelligence (2014) the ABRS market in mobile context will grow from 1.6 billion $\$$, in 2014 , to 34.6 billion $\$$ in 2020 , each year, about 800 billion transactions requiring different levels of biometric authentication.

A Facial Recognition System (FRS) is a computer application capable of identifying an individual from a digital image or video frame.

Face recognition represents a non-intrusive and user-friendly biometric security technique (it is the least invasive method); thus, it has a variety of potential applications in public security, law enforcement, e-commerce, access control, information security and video surveillance.

Facial biometrics have been integrated with consumer electronics in order to facilitate authentication, replacing the usage of passwords. Using data from cameras, a computer / mobile phone can be unlocked when the user stands in front of it.

$\boldsymbol{F R S}$ solutions can be used to authenticate the right of electronic access to Collaborative Decision Support Systems (CDSSs). Under the influence of the new technologies, CDSSs have evolved from few people located in a "decision room" to an unlimited number of participants over a distributed architecture (Filip, Zamfirescu $\&$ Ciurea, 2017). Thus, the identification of the participants has become an important topic.

Face recognition combined with Artificial Intelligence $(A I)$ is also set to change the face of the Advertising Sector. The retailer Tesco intends to install hi-tech OptimEyes (Amscreen, 2018) screens in order to deliver targeted ads to customers after identifying the age range and gender of the waiting customers.

The biggest problem of automatic facial recognition systems is that, the recognition rate is heavily affected by changes in illumination, pose and facial expressions. So, it is difficult to design a system sufficiently simple to handle all these problems.

The most popular global facial feature extraction methods are: Principal Component Analysis (PCA), Linear Discriminant Analysis (LDA), Independent Component Analysis (ICA), Gabor 
filters combined with Local binary pattern analysis (Lei et al., 2011), Gabor Wavelet (Zhou $\&$ Wei, 2006) (Vinay \& Shreyas, 2006).

Zhou \& Wei (2006) presented a new facial feature extraction algorithm based on Gabor wavelets and AdaBoost. Vinay \& Shreyas (2006) described the usage of Gabor wavelets for efficient face representation.

Li, Gao \& Wang (2017) presented a face recognition algorithm based on auto-encoder networks with dropout. Using a 4-layer network with 784-800-800-40 neuron structure, the system achieved a $97.5 \%$ recognition rate on the ORL (Samaria \& Harter, 1994) dataset, when $90 \%$ of the data was used for training and $10 \%$ for testing.

In many computer vision and image processing applications, the algorithms based on features constructed from responses of Gabor filters, also known as Gabor features, are among the top performers. Some top biometric systems based on Gabor features are: Daugman's (Daugman J. G., 1993) iris recognition system, Jain's (Jain, Chen \& Demirkus, 2007) fingerprint matching algorithm, face recognition systems (Messer et al., 2004).

Gabor features extract local pieces of information which are then combined to recognize an object or a region of interest.

In this paper, a new face recognition algorithm that combines Deep Learning techniques with multi-level Gabor features is proposed.

First, for each face image the Gabor features are extracted using a multi-level approach. Then, the user's face model is generated using Deep Learning techniques. In the recognition phase, the classifier compares the feature vectors of a sample image with the facial models that are learned during training, and selects the model with the maximum likelihood value.

The rest of paper is organized as follows: a short review of the Gabor wavelet filter is described in Section 2. In Section 3, the Neural Networks used for subject modelling are presented. The proposed algorithm is described in Section 4. In Section 5, experimental results are shown. Finally, the conclusions are presented in Section 6.

\section{Gabor Wavelet Filters}

First introduced in the 40 s by the Hungarianborn engineer Denis Gabor (Gabor, 1946), the Gabor functions or atoms have been used in many different fields such: audio signal processing, image compression, edge detection, filter design, object recognition.

The Gabor Wavelet Filter represents a band-pass linear filter whose impulse response is defined by a harmonic function multiplied by a Gaussian function. Basically, it consists of a group of wavelets; each wavelet captures the energy at a specific frequency and in a specific direction. In image processing, Gabor Wavelet filters are used for features extraction in texture-based image analysis. This means that it basically analyses whether there is any specific frequency content in the image in a specific direction in a localized region around the point or region of analysis. These Gabor wavelets provide a complete image representation (Lee, 1996).

Marceelja (1980) and Daugman (1985) advanced the idea that simple cells in the visual cortex of mammalian brains can be modelled using Gabor functions. Thus, image analysis using Gabor filters is thought to be similar to perception in the human visual system. By presenting optimal localization properties in both spatial and frequency domain and thus, are well suited for texture segmentation, edge detection and image representation problems (Weldon, Higgins, \& Dunn, 1996).

A bi-dimensional Gabor filter, $G_{f, \theta}(x, y)$ can be viewed as a Gaussian kernel function modulated by a sinusoidal plane wave of a particular frequency and orientation as follows:

$G_{f, \theta}(x, y)=e^{-\left[\frac{x_{\theta_{n}}^{2}}{\sigma_{x}^{2}}+\frac{y_{\theta_{n}}^{2}}{\sigma_{y}^{2}}\right.} e^{i\left(2 \pi f x_{\theta_{n}}+\varphi\right)}$

where, $x_{\theta_{n}}=x \cos \theta_{n}+y \sin \theta_{n}$,

$y_{\theta_{n}}=y \cos \theta_{n}-x \sin \theta_{n}, f$ represents the central frequency of the sinusoidal wave, $\varphi$ the phase, $\theta_{n}$ the orientation of the wave plane and $\sigma_{x} / \sigma_{y}$ represent the standard deviations of the Gaussian envelope along the $X / Y$ axes. 
The filter has two components (Figure 1), a real and an imaginary component, representing the orthogonal directions:

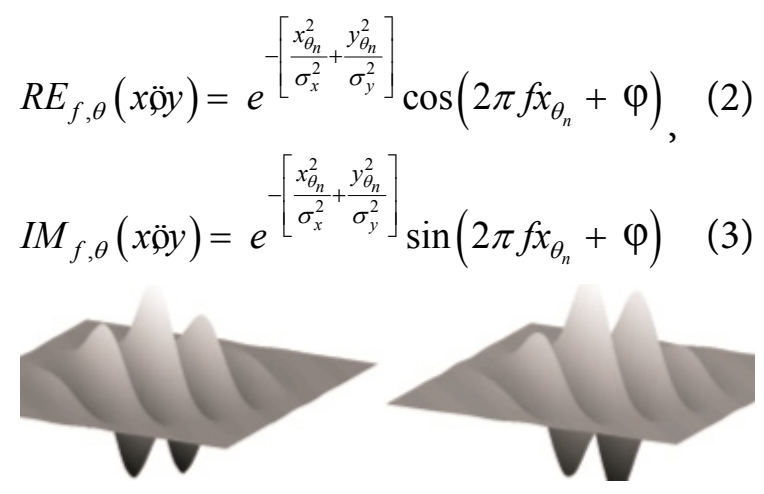

Figure 1. Example of Gabor filter (real and imaginary part) (C) (Štruc \& Pavešić, 2010)

The $2 D$ filters defined by relation (1), represent a group of wavelets and can optimally capture both local orientation and frequency information from an image. By using $G_{f, \theta}(x, y)$, the image is filtered at various orientations, frequencies and standard deviations. Thus, in order to design a Gabor filter, the phase, the orientations, the frequencies and the standard deviations must be defined.

The phase is set to $\varphi=\frac{\pi}{2}$ and the angle $\theta_{n}$ is
defined by: $\theta_{n}=\frac{\pi}{p}(n-1)$

where, $p$ denotes the number of orientations and $n \in\{1 \ldots p\}$.

In this paper the real part of the Gabor representation is used for feature extraction. Thus, the $2 D$ Gabor filter bank is defined by equations (2) and (4).

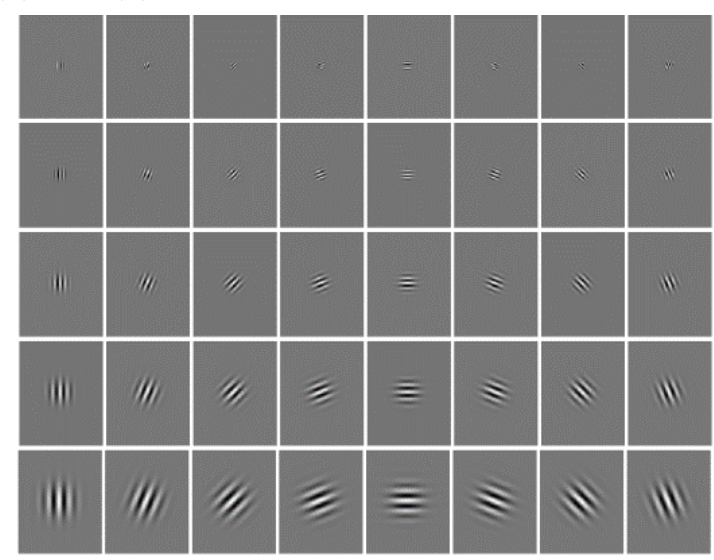

Figure 2. Real part of the used Gabor filters (C) (̌̌truc \& Pavešić, 2010)
The most important step in designing Gabor filters is the selection of the filter parameters. This research uses a 40 channels (Figure 2) filter bank consisting of eight orientations $\theta \in\left\{0, \frac{\pi}{8}, \frac{2 \pi}{8}, \frac{3 \pi}{8}, \frac{4 \pi}{8}, \frac{5 \pi}{8}, \frac{6 \pi}{8}, \frac{7 \pi}{8}\right\}$, five spatial frequencies $f \in\left\{\frac{1}{4}, \frac{1}{4 \sqrt{2}}, \frac{1}{8}, \frac{1}{8 \sqrt{2}}, \frac{1}{16}\right\}, \quad$ and variance values $\sigma_{x}=\sqrt{2} / f, \sigma_{y}=\sqrt{2} / f$.

The Gabor feature representation of a grey-scale image $I(x, y) \in R^{m \times n}$, where $m \times n$ represents the image size in pixels, is obtained by convolving the input face image with the created Gabor filters (Figure 3).

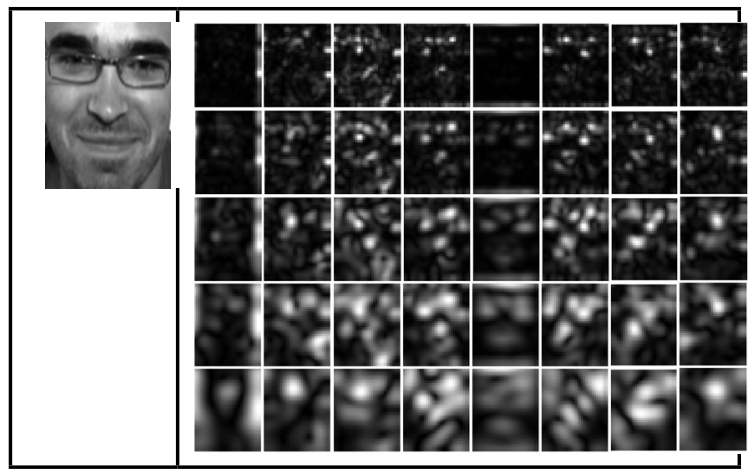

Figure 3. Gabor filter output

\section{Deep Learning Using Auto-encoders}

Normally, the neural networks used for classification are trained to map an input vector to an output that represents the known classification classes. An alternative approach is to use autoassociative neural networks, also called autoencoders or Diabolo networks in order to learn a model of each class.

An auto-encoder, auto-associator or Diabolo network (Bengio, 2009) is an artificial neural network used for unsupervised learning of efficient codings (Liou, Huang, \& Yang, 2008) (Liou et al., 2014). Typically, the aim of an auto-encoder is to generate (learn) a compressed representation (also known as encoding) for a dataset, with the scope to obtain a dimensionality reduction. This initial concept has evolved, and auto-encoders have become widely used for learning generative models of data. 


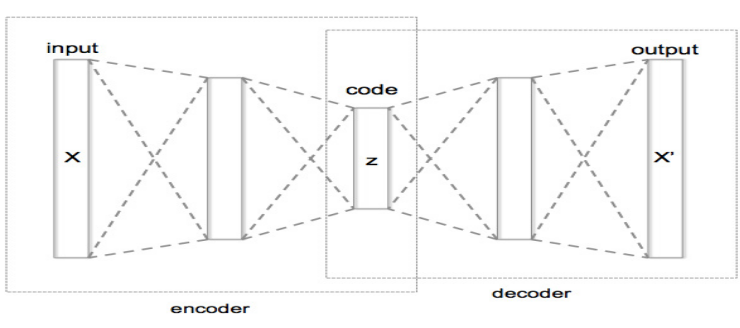

Figure 4. Schematic structure of an autoencoder with 3 fully-connected hidden layers

Figure 4 illustrates the basic architecture of an auto-encoder. From the architectural point of view, the simplest form of an auto-encoder is a feedforward, non-recurrent neural network that is very similar to the multilayer perceptron $(\boldsymbol{M L P})$. It consists of an input layer, an output layer and one or more hidden layers connecting them. The main difference between a MLP and a Diabolo network consists in the number of nodes contained in the output layer. In the case of Diabolo networks the output layer has the same number of nodes as the input layer.

The training of an auto-encoder is accomplished by using one of the many backpropagation algorithms (conjugate gradient method, steepest descent, etc.), with the purpose that the network reconstructs its own inputs.

An auto-encoder always consists of two parts, an encoder and a decoder. The encoder and decoder can have multiple layers, but for simplicity each of them has only one layer. Being given an input $x \in \mathbb{R}^{D}$, the encoder will map it onto another vector $z \in \mathbb{R}^{P}$ as follows:

$z=\sigma_{1}(W x+b)$,

where: $\sigma_{1}$ is an element-wise activation function (transfer function) for the encoder, $W \in \mathbb{R}^{D \times P}$ is a weight matrix and $b \in \mathbb{R}^{P}$ is a bias vector.

By using the decoder, the encoded representation $z$ is mapped onto the reconstruction vector $x^{\prime}$, an estimate of the original input vector $x$.

$x^{\prime}=\sigma_{2}\left(W^{\prime} z+b^{\prime}\right)$,

where: $\sigma_{2}$ is the transfer function for the decoder, $W^{\prime} \in \mathbb{R}^{D \times P}$ is a weight matrix and $b^{\prime} \in \mathbb{R}^{D}$ is a bias vector.
Because an auto-encoder is designed to replicate the input data to the outputs, the biggest risk is that it will learn the identity function, the trivial case. In order to prevent this situation, different constraints can be placed on the network. The simplest one consists in limiting the number of hidden neurons: e.g. if the input vector has 100 elements, then the hidden layer has 50 elements. With this constraint the encoder will compress the 100 -element vector into a 50 -element vector and the decompression phase will expand the 50 -element vector into a 100 -element vector that is ideally close to the original input. This simple auto-encoder often ends up learning a low-dimensional representation of the inputs very similar to $\boldsymbol{P C A}$ (in the case that the input data is correlated).

Another type of constraint, more often used, is the "sparsity constraint" or "sparsity parameter". In this case the hidden layer has a large number of neurons (compared with the number of inputs), but for each input only a small number of them will be activated (it produces an activation value closer to " 1 ") during the training of the network. Such encoders are called "sparse auto-encoder" (Olshausen \& Field, 1997) and they produce a sparse representation of the inputs. The "sparsity parameter" is a small value close to zero (e.g. $0,05)$ and it represents the average activation value, for any hidden node, over all the training samples.

\section{The Proposed Algorithm}

The algorithm proposed in this paper (presented in Figure 5) consists of the following stages: multi-level feature vectors extraction using Gabor filters, input data model generation using auto-encoders and unknown subject's identification. The algorithm is designed to use grey-scale images.

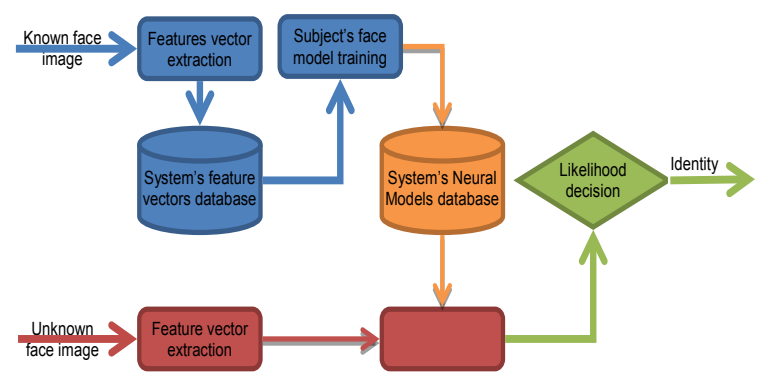

Figure 5. Block diagram of the proposed face recognition procedure 
The "Feature Vector Extraction" phase generates the feature vector from each known subject image using Gabor filters in a multi-level approach (Figure 6).

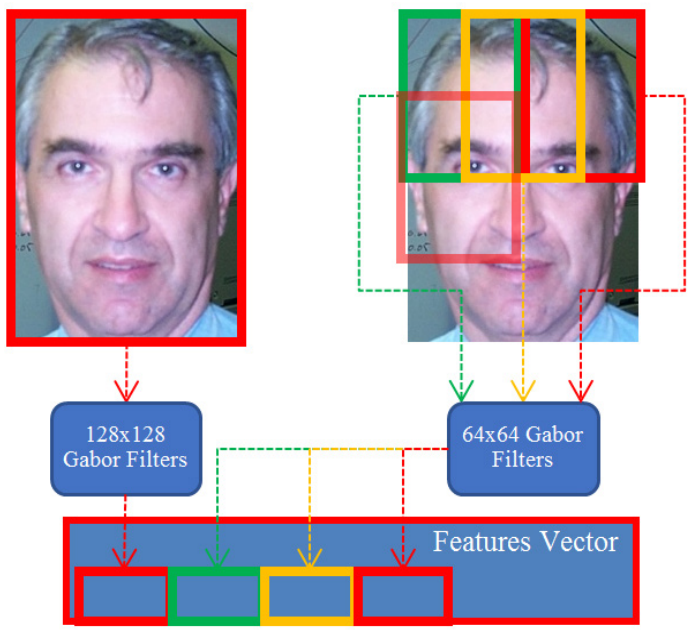

Figure 6. Feature Vector Extraction

First, the image is converted to grey-scale, if necessary, by converting the $\boldsymbol{R} \boldsymbol{G B}$ (red - green - blue) colour space to $\boldsymbol{H S V}$ (hue - saturation value) colour space and the keeping only the $\boldsymbol{V}$; and afterwards, is resized to $128 \times 128$ pixels.

From the resulted image the first part of the feature vector is extracted by applying the first set of Gabor filters. A total of 40 outputs are obtained, each output with the size of $128 \times 128$ pixels. In order to overcome the dimensionality issue, the outputs are down-sampled by a factor of $6 x 6$, superimposing over the image a rectangular sampling grid and retaining only the values located under the sampling grid's nodes. And finally, the down-sampled outputs are normalized in order to have zero mean and unit variance.

For the second part of the feature vector extraction, the image is divided into blocks of $64 x 64$ pixels with an overlap between the blocks of 32 pixels. Each image block is then convoluted with the second Gabor filter bank; the resulted output is down-sampled with a factor of $6 x 6$ and normalized. The final feature vector consists of 53640 elements, calculated as follows:

$40 \times\left\lfloor\frac{128}{6}\right\rfloor \times\left\lfloor\frac{128}{6}\right\rfloor+9 \times 40 \times\left\lfloor\frac{64}{6}\right\rfloor \times\left\lfloor\frac{64}{6}\right\rfloor$,

Both filter banks have the same orientations and frequencies. The difference between them is given by the size of the used filters: while the first bank uses filters of $128 \times 128$, the second filter bank has a size of $64 x 64$.
By applying a two-stage feature extraction technique, the resulted feature vector contains two types of features: full-face features and local features. The first Gabor filter bank generates a full-face presentation of the subject, while the second Gabor filter bank is focused on extracting relevant information around major facial features (such as eyes, mouth, nose).

The second step of this algorithm consists in "Data Model generation" with the help of a stacked neural network architecture combining a Diabolo network and a SoftMax layer (Figure 7). In the first step the auto-encoder network is trained using the feature vectors extracted in the previous step. The hidden layer of the autoencoder will map the input data to a reduced subspace that represents the compressed version of the input. For training the auto-encoder, the selected algorithm is the scaled conjugate gradient descent (SCG) backpropagation.

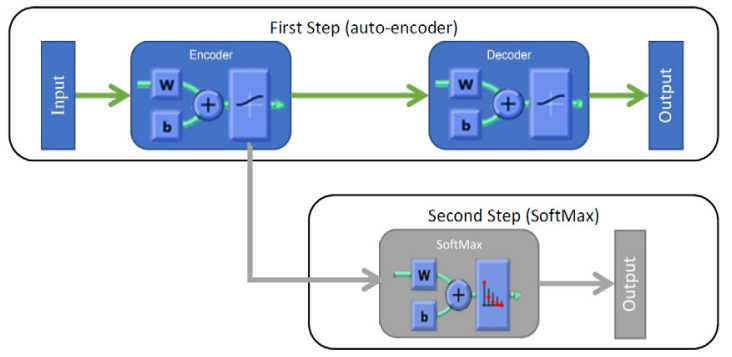

Figure 7. Stacked Architecture

The encoder of the trained network is used to generate the feature vectors for the final step of the algorithm. In this stage a SoftMax network is trained to classify the compressed feature vectors into different subject classes. The number of the output classes of the SoftMax layer is equal to the number of the known subjects.

\section{Experimental Results}

All experiments were performed using one colour face database Caltech 101 (Weber, 1999) and three grey-scale face databases Yale Face (Bellhumer, Hespanha \& Kriegman, 1997), Extended Yale B (Georghiades, Belhumeur, \& Kriegman, 2001) and ORL (Samaria \& Harter, 1994). The Caltech database contained 450 frontal face colour images ( $896 \times 592$ pixels) of 26 unique subjects with different lighting, expressions and backgrounds (Figure 8). The face regions from the images were extracted using a Viola-Jones (Viola \& Jones, 2001) face detector. Some images 
had very poor lighting conditions (were too dark or too bright) and were excluded. After the face segmentation, more than 200 faces of 26 unique individuals remained.

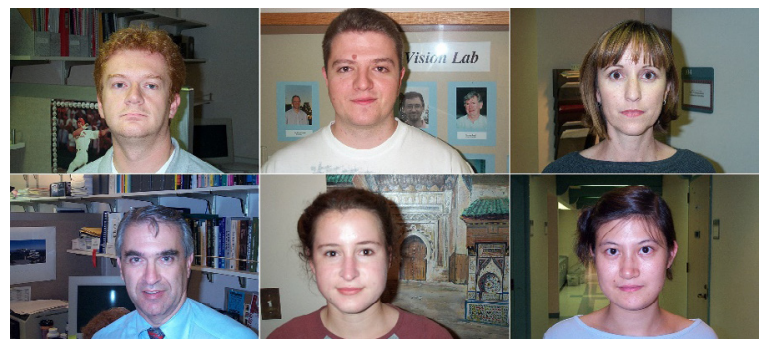

Figure 8. Sample images from Caltech database

The Yale Face database consisted of 11 different grey-scale pictures of 15 unique individuals. The images were acquired with several configurations and facial expressions: normal, centre-light, with glasses, happy, left-light, no glasses, right-light, sad, sleepy, surprised, and wink (Figure 9).

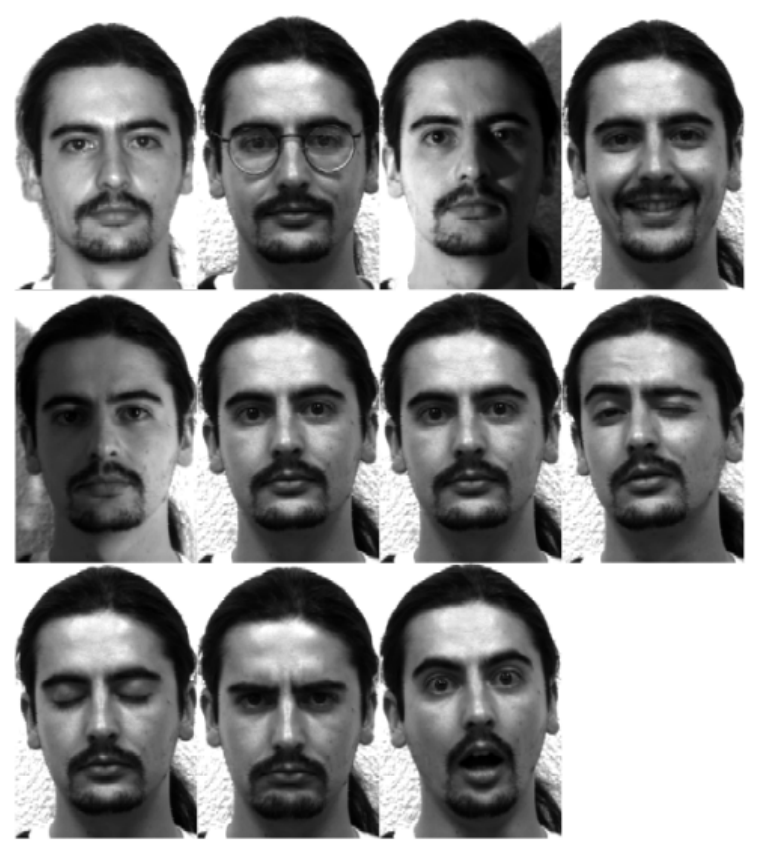

Figure 9. Sample images from Yale database

The extended Yale Face Database B contained 16128 grey-scale images of 28 human subjects under 9 poses and 64 illumination conditions, resulting 576 viewing conditions for every subject. The images were aligned, cropped and resized. The nine poses recorded were as follows: pose 0 was the frontal pose; poses 1, 2, 3, 4, and 5 were about 12 degrees from the camera optical axis, while poses 6,7 , and 8 were about 24 degrees. In this paper, only a subset of the database was used, consisting of 63 pictures per subject under the following conditions: nine poses ( 0 to 8 ), five azimuth positions $(0,+0.5,-0.5,+1,-1$ degrees $)$ and three elevation positions $(0,+10,-10$ degrees $)$ (see Figure 10).

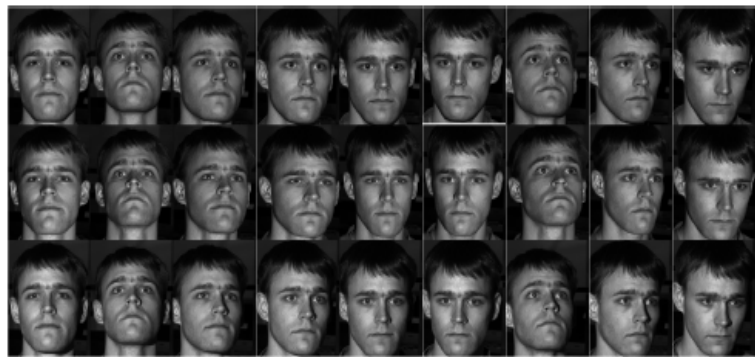

Figure 10. Sample images from Yale B database

The ORL (Olivetti Research Laboratory) face database contained a set of grey-scale images of faces taken between April 1992 and April 1994. There were 10 different images of 40 distinct subjects. Figure 11 illustrates some of the images from the database. The images were taken at different times, varying slightly, with different facial expressions (open / closed eyes, smiling / non-smiling) and facial details (glasses/ no-glasses). All the images were taken against a dark homogeneous background and the subjects were in up-right, frontal position.
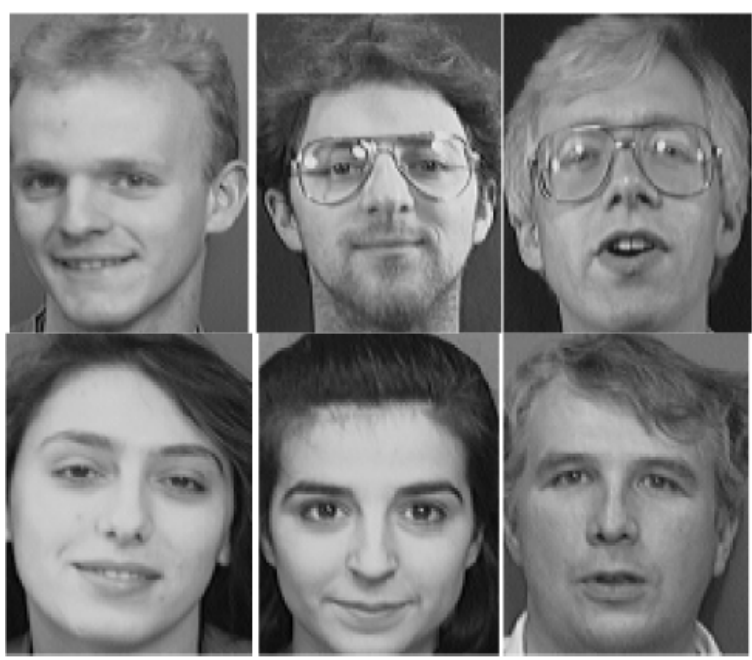

Figure 11. Sample images from ORL database

By running the experiments with different configuration parameters and measuring the error rates, the optimal neural network configuration parameters were determined. The corresponding values are as follows:

\section{- $\quad$ L2 Weight Regularization 0.04}

$\begin{array}{lll}\text { - } & \text { Sparsity Regularization } & 1.6 \\ \text { - } & \text { Sparsity Proportion } & 0.1 \\ \text { - Hidden Layer Size } & 200\end{array}$

https://www.sic.ici.ro 
In the experiments, the encoder used the positive saturating linear transfer function, given by:

$f(z)= \begin{cases}0 & , z \leq 0 \\ z & , 0<z<1 \\ 1 & , z \geq 1\end{cases}$

While the decoder transfer function was set to pure linear:

$$
f(z)=z,
$$

For the testing of this algorithm, all the available subjects were used, and the databases were divided in two: the first part, containing 4 pictures per each subject, was used to generate the face model; the second part consisting of the remaining pictures for each person was used as test data. With the given setup, the effectiveness of the proposed algorithm was measured by using several standard error and recognition rates, as follows:

- the first rate is the rank-one recognition rate $(\boldsymbol{R O R})$ - it means that the nearest neighbour is an image of the same person;

- the false acceptance rate $(\boldsymbol{F A R})$ indicates the percentage of accepted non-authorized users;

- the false rejection rate $(\boldsymbol{F R R})$ indicates the percentage of incorrectly rejected authorized users;

- the minimal half total error rate (mHTER):

$$
\boldsymbol{m H T E R}=\min \left(\frac{F A R+F R R}{2}\right),
$$

During the recognition experiments that are performed on the test data, a similarity matrix (Figure 12) is produced from the scores generated by the SoftMax layer. For $M$ test images and $N$ subjects in the database, the corresponding similarity matrix has a size of $N \times M$.

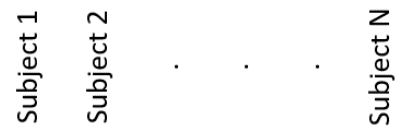

$$
\begin{aligned}
& \begin{array}{c}
\text { Image } 1 \text { (Subject 1) } \\
\text { Image } 2 \text { (Subject 1) } \\
\text { Image M (Subject N) }
\end{array}\left[\begin{array}{cccccc}
1 & 0.44 & . & . & . & 0.3 \\
0.9 & 0.2 & . & . & . & 0.1 \\
. & . & . & . & . & . \\
. & . & . & . & . & . \\
. & . & . & . & . & . \\
0.3 & 0.2 & . & . & . & 1
\end{array}\right]
\end{aligned}
$$

Figure 12. Visualization of a similarity matrix $($ size $N \times M)$
The $\boldsymbol{F A R}$ and $\boldsymbol{F R} \boldsymbol{R}$ values are calculated by imposing different decision threshold values $(\Delta)$ on the similarity matrix (e.g. image $i$ corresponding to subject $j$ will be counted as impostor if Score $_{i, x}>\Delta$ where $1 \leq x \leq N$ and $x \neq j$ ).

In order to measure and prove the efficiency of the algorithm proposed in this paper, four different classical face recognition algorithms were trained and tested, using the same data set (Štruc \& Pavešić, 2009) (Štruc \& Pavešić, 2010):

- Gabor - PCA and the nearest neighbour classifier;

- Gabor - LDA and the nearest neighbour classifier;

- Gabor - KFA (Kernel Fisher Analysis) and the nearest neighbour classifier;

- Gabor - KPCA (Kernel Principal Component Analysis) and the nearest neighbour classifier.

The experimental results are presented in Tables 1 - 4.

Table 1. Recognition rate comparison

\begin{tabular}{|l|c|c|c|c|c|}
\hline & Caltech & ORL & Yale & Yale B & Mean \\
\hline $\begin{array}{l}\text { Own } \\
\text { algorithm }\end{array}$ & $95.00 \%$ & $97.14 \%$ & $91.83 \%$ & $98.20 \%$ & $95.54 \%$ \\
\hline PCA & $84.58 \%$ & $93.33 \%$ & $81.93 \%$ & $96.40 \%$ & $89.06 \%$ \\
\hline LDA & $98.33 \%$ & $98.10 \%$ & $97.36 \%$ & $99.10 \%$ & $98.22 \%$ \\
\hline KFA & $98.33 \%$ & $96.19 \%$ & $96.31 \%$ & $99.40 \%$ & $97.56 \%$ \\
\hline KPCA & $82.92 \%$ & $95.24 \%$ & $84.57 \%$ & $94.89 \%$ & $89.41 \%$ \\
\hline
\end{tabular}

Table 2. mHTER comparison

\begin{tabular}{|l|c|c|c|c|c|}
\hline & Caltech & ORL & Yale & Yale B & Mean \\
\hline $\begin{array}{l}\text { Own } \\
\text { algorithm }\end{array}$ & $0.89 \%$ & $0.37 \%$ & $2.43 \%$ & $0.11 \%$ & $0.95 \%$ \\
\hline PCA & $3.04 \%$ & $1.80 \%$ & $6.17 \%$ & $1.66 \%$ & $3.17 \%$ \\
\hline LDA & $0.44 \%$ & $0.14 \%$ & $0.92 \%$ & $0.13 \%$ & $0.41 \%$ \\
\hline KFA & $0.92 \%$ & $1.97 \%$ & $2.04 \%$ & $0.34 \%$ & $1.32 \%$ \\
\hline KPCA & $3.18 \%$ & $1.09 \%$ & $4.68 \%$ & $1.34 \%$ & $2.57 \%$ \\
\hline
\end{tabular}


Table 3. FAR at mHTER comparison

\begin{tabular}{|l|c|c|c|c|c|}
\hline & Caltech & ORL & Yale & Yale B & Mean \\
\hline $\begin{array}{l}\text { Own } \\
\text { algorithm }\end{array}$ & $1.37 \%$ & $0.75 \%$ & $1.73 \%$ & $0.23 \%$ & $1.02 \%$ \\
\hline PCA & $3.16 \%$ & $0.75 \%$ & $2.99 \%$ & $1.53 \%$ & $2.11 \%$ \\
\hline LDA & $0.47 \%$ & $0.27 \%$ & $0.92 \%$ & $0.26 \%$ & $0.48 \%$ \\
\hline KFA & $1.42 \%$ & $1.09 \%$ & $1.62 \%$ & $0.67 \%$ & $1.20 \%$ \\
\hline KPCA & $3.87 \%$ & $2.18 \%$ & $3.77 \%$ & $2.08 \%$ & $2.98 \%$ \\
\hline
\end{tabular}

Table 4. FRR at mHTER comparison

\begin{tabular}{|l|c|c|c|c|c|}
\hline & Caltech & ORL & Yale & Yale B & Mean \\
\hline $\begin{array}{l}\text { Own } \\
\text { algorithm }\end{array}$ & $0.42 \%$ & $0.00 \%$ & $3.13 \%$ & $0.00 \%$ & $0.89 \%$ \\
\hline PCA & $2.92 \%$ & $2.86 \%$ & $9.34 \%$ & $1.80 \%$ & $4.23 \%$ \\
\hline LDA & $0.42 \%$ & $0.00 \%$ & $0.92 \%$ & $0.00 \%$ & $0.34 \%$ \\
\hline KFA & $0.42 \%$ & $2.86 \%$ & $2.46 \%$ & $0.00 \%$ & $1.44 \%$ \\
\hline KPCA & $2.50 \%$ & $0.00 \%$ & $5.59 \%$ & $0.60 \%$ & $2.17 \%$ \\
\hline
\end{tabular}

\section{Conclusion}

In this paper, a novel hybrid algorithm for face identification / recognition was presented by combining Gabor-based features with Deep Learning methods. The two-stage feature extraction technique allows for both full-face features and local features to be used in the training and recognition stages.

Comparing the average recognition rate $(\boldsymbol{R} \boldsymbol{O R}$

- Table 1), the system analysed in the article outperforms two of the classical face recognition algorithms. In comparison with the other ones the difference is less than $\mathbf{2 \%}$, thus proving the effectiveness of the proposed algorithm.

By analysing the results presented in Table 1, it can be observed that the classical face recognition algorithms are heavily influenced by the different

\section{REFERENCES}

1. Acuity Market Intelligence. (2014). The Global Biometrics and Mobility Report: The Convergence of Commerce and Privacy Market Analysis and Forecasts 2014-2020.

2. Amscreen (2018). Amscreen to launch OptimEyes advertising platform. Available at: $\quad h t t p: / / w w w . a m s c r e e n . e u / a m s c r e e n-t o-$ launch-optimeyes-advertising-platform-2/ facial expressions. In the case of the classic approach, the recognition rate drops (in some cases) by as much as $\mathbf{1 2 \%}$, from $\mathbf{9 5 . 2 4 \%}$ till 82.92\%. The new hybrid algorithm presented a degradation smaller than $\mathbf{7 \%}$.

Being given a small number of training images per subject (4 images were used for training), the proposed system managed to achieve a recognition rate over 95\%. In the case of "False Acceptance Rate" (Table 3), the new method obtained a $\mathbf{1 . 0 2} \%$ FAR, making it an ideal candidate for face verification systems.

When compared to the auto-encoder architecture presented by Li et al. (2017), the proposed hybrid architecture offers, on the ORL dataset, almost the same recognition rate $\mathbf{( 9 7 . 1 4 \%}$ vs. $\mathbf{9 7 . 5 \%})$ having a simpler structure (one layer vs. four layers), using a smaller number of training images and a higher number of test images.

During the experiments presented in this paper, the system configuration consisting of the image size, down-sample rate, and total number of neurons was determined by the trial and error methods. These experiments must be continued, in order to determine the optimal number of neurons contained in the network, and also to check if a correlation exists between the number of neurons and the number of subjects. Also, the system must be tested using different image sizes and multiple decomposition levels.

The experiments were carried out using MATLAB $^{\circledR}$ and the PhD Toolbox (Štruc \& Pavešić, 2010) on an PC with 48GB of RAM, Intel Core i7 8700K CPU and GeForce GTX 1080 GPU with CUDA capabilities.

3. Bellhumer, P. N., Hespanha, J. P. \& Kriegman, D. J. (1997). Eigenfaces vs. fisherfaces: Recognition using class specific linear projection, IEEE Transactions on Pattern Analysis and Machine Intelligence, 17(7), 711-720.

4. Bengio, Y. (2009). Learning Deep Architectures for AI, Foundations and Trends ${ }^{\circledR}$ in Machine Learning, 2(1), 1-127. 
5. Daugman, J. G. (1985). Uncertainty relation for resolution in space, spatial frequency, and orientation optimized by two-dimensional visual cortical filters, Journal of the Optical Society of America, 2(7), 1160-1169.

6. Daugman, J. G. (1993). High confidence visual recognition of persons by a test of statistical independence, IEEE Transactions on Pattern Analysis and Machine Intelligence, 15(11), 1148-1161.

7. Filip, F. G., Zamfirescu, C. B. \& Ciurea, C. (2017). Computer-Supported Collaborative Decision-Making. Springer International Publishing.

8. Gabor, D. (1946). Theory of communication, Journal of the Institution of Electrical Engineers - Part III: Radio and Communication Engineering, 93(22), 429-457.

9. Georghiades, A. S., Belhumeur, P. N. \& Kriegman, D. J. (2001). From Few to Many: Illumination Cone Models for Face Recognition under Variable Lighting and Pose, IEEE Transactions on Pattern Analysis and Machine Intelligence, 23(6), 643-660.

10. Jain, A., Chen, Y. \& Demirkus, M. (2007). Pores and ridges: Fingerprint matching using level 3 features, IEEE Transactions on Pattern Analysis and Machine Intelligence, 29(1), 15-27.

11. Lee, T. S. (1996). Image representation using 2D Gabor wavelets, IEEE Transactions on Pattern Analysis and Machine Intelligence, 18(10), 959-971.

12. Lei, Z., Liao, S., Pietikäinen, M. \& Li, S. Z. (2011). Face recognition by exploring information jointly in space, scale and orientation, IEEE Transactions on Image Processing, 20(1), 247 - 256.

13. Li, F., Gao, X. \& Wang, L. (2017). Face Recognition Based on Deep Autoencoder Networks with Dropout. In 2017 2nd International Conference on Modelling, Simulation and Applied Mathematics Advances in Intelligent Systems Research, 32 (pp. 243-247).
14. Liou, C.-Y., Cheng, W.-C., Liou, J.-W. \& Liou, D.-R. (2014). Autoencoder for words, Neurocomputing, 139, 84-96.

15. Liou, C.-Y., Huang, J.-C. \& Yang, W.-C. (2008). Modeling word perception using the Elman network, Neurocomputing, 71(16-18), 3150-3157.

16. Marĉelja, S. (1980). Mathematical description of the responses of simple cortical cells, Journal of the Optical Society of America, 70(11), 1297-1300. DOI:10.1364/JOSA.70.001297

17. Messer, K., Kittle, J., Sadegh, M., Hamouz, M., \& Kosti, A. (2004). Face authentication test on the BANCA database. In Proceedings of the 17th International Conference on Pattern Recognition(ICPR), 4 (pp. 523-532). DOI: 10.1109/ICPR.2004.1333826

18. Olshausen, B. A. \& Field, D. J. (1997). Sparse coding with an overcomplete basis set: A strategy employed by V1?, Vision Research, 37(23), 3311-3325.

19. Samaria, F. S. \& Harter, A. (1994). Parameterisation of a stochastic model for human face identification. In Proceedings of 2nd IEEE Workshop on Applications of Computer Vision (pp. 138-142). DOI:10.1109/ACV.1994.341300

20. Štruc, V. \& Pavešić, N. (2009). GaborBased Kernel Partial-Least-Squares Discrimination Features for Face Recognition, Informatica, 20(1), 115-138.

21. Štruc, V. \& Pavešić, N. (2010). The Complete Gabor-Fisher Classifier for Robust Face Recognition. In EURASIP Journal on Advances in Signal Processing. DOI:10.1155/2010/847680

22. Vinay, K. B. \& Shreyas, B. S. (2006). Face Recognition Using Gabor Wavelets. In Fortieth Asilomar Conference on Signals, Systems and Computers (pp. 593-597). IEEE, Pacific Grove, CA, USA.

23. Viola, P. \& Jones, M. (2001). Rapid object detection using a boosted cascade of 
simple features. In Proceedings of 2001 IEEE Computer Society Conference on Computer Vision and Pattern Recognition, 1 (pp. 511-518).

24. Weber, M. (1999). A frontal face dataset collected by Markus Weber at California Institute of Technology. Available at: http://www.vision.caltech.edu/Image Datasets/faces/faces.tar
25. Weldon, T. P., Higgins, W. E. \& Dunn, D. F. (1996). Gabor filter design for multiple texture segmentation, Optical Engineering, 35(10), 2852-2863.

26. Zhou, M. \& Wei, H. (2006). Face Verification Using Gabor Wavelets and AdaBoost. In 18th International Conference on Pattern Recognition, 1 (pp. 404-407). IEEE, Hong Kong, China DOI:10.1109/ICPR.2006.536 